\title{
Automated breast volume scanning combined with shear wave elastography for diagnosis of triple-negative breast cancer and human epidermal growth factor receptor 2-positive breast cancer

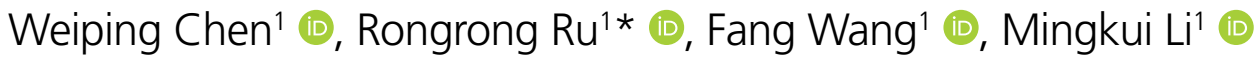

\section{SUMMARY}

OBJECTIVE: To explore the values of automated breast volume scanning (ABVS) combined with shear wave elastography (SWE) in the differential diagnosis of triple-negative breast cancer (TNBC) and human epidermal growth factor receptor 2-positive breast cancers (HER2+BC). METHODS: In this study, 28 patients with TNBC and 32 patients with HER2+BC were enrolled. The characteristics of ABVS and virtual touch quantification (VTQ) in SWE of all patients were reviewed. The multivariate logistic regression analysis was carried out and the receiver operating characteristic curves of ABVS and ABVS+VTQ were drawn.

RESULTS: In ABVS imaging, the microcalcification, posterior echo, internal echo, shape, and edge had significant difference between TNBC and HER2+BC groups $(p<0.05)$. The regular shape was the independent factor for TNBC $(p=0.04$, odds ratio [OR] $=4.479)$, and the microcalcification in mass was the independent factor for HER2+BC ( $p=0.01, O R=2.997)$. In VTQ imaging, the shear wave velocity (SWV) max' $^{\prime} S W V_{\text {min' }^{\prime}}$ and SWV $V_{\text {mean }}$ in TNBC group were significantly lower than those in HER2+BC group $(p<0.001)$. The sensitivity, specificity, and accuracy of $A B V S+V T Q$ in diagnosing TNBC were higher than those of ABVS alone.

CONCLUSIONS: ABVS combined with SWE has certain advantages in differentiating TNBC from HER2+BC, which is helpful for the treatment planning and prognosis judgment.

KEYWORDS: Breast cancer. Ultrasound. Elasticity imaging techniques. Efficiency.

\section{INTRODUCTION}

Breast cancer is a malignant tumor in women, causing the highest morbidity and mortality ${ }^{1}$. Triple-negative breast cancer (TNBC) is a particular type of breast cancer that is characterized by its biological aggressiveness, worse prognosis, and lack of prognostic markers or therapeutic targets in contrast with hormonal receptor-positive breast cancer and human epidermal growth factor receptor 2-positive breast cancer $(\mathrm{HER} 2+\mathrm{BC})^{2}$. Both TNBC and HER2+BC are considered to be the most immunogenic breast cancers and are highly heterogeneous ${ }^{3}$. HER2 + BC is sensitive to the targeted molecular therapy, though its prognosis is poor. However, TNBC has high invasiveness and lacks the targeted standard treatment, so its prognosis is worse than HER2+BC 4 . The majority of patients with TNBC die in the first 5 years after treatment ${ }^{5}$. Therefore, improving the diagnostic accuracy of TNBC and HER2+BC plays an important role for the treatment choice and prognosis. Ultrasound is the most common method of breast examination. Automated breast volume scanning (ABVS) is a three-dimensional breast ultrasound technology, which has the advantage of multiplanar reconstruction ${ }^{6}$. Compared with handheld ultrasound, ABUS can overcome the dependence on operators

\footnotetext{
${ }^{1}$ Hangzhou Normal University, Xiaoshan Hospital, Department of Ultrasound - Hangzhou, China.

*Corresponding author: rongrongru@21cn.com

Conflicts of interest: the authors declare there is no conflicts of interest. Funding: none.

Received on June 13, 2021. Accepted on July 06, 2021.
} 
and improve the repeatability of inspection. In addition, it can show the structural distortion in coronal plane ${ }^{7}$. Shear wave elastography (SWE) is a new imaging technology based on ultrasonic imaging that can provide the tissue stiffness information $^{8}$. It has the noninvasive, simple, and real-time advantages ${ }^{8}$. In clinics, SWE is used to measure the elastic modulus of tissue and reflect the stiffness properties, contributing to the early diagnosis and assessment of disease? ${ }^{9}$. This study investigated the values of ABVS combined with SWE in the diagnosis of TNBC and HER2+BC, for providing more information for effective diagnosis of breast cancer.

\section{METHODS}

\section{Subjects}

Sixty female patients with TNBC or HER $2+B C$ confirmed by surgery and pathology in our hospital from July 2017 to January 2020 were enrolled in this study. There were a total of 60 lesions. The lesion size was $1.3-3.6 \mathrm{~cm}$, with average size of $2.3 \pm 1.4 \mathrm{~cm}$. Out of these 60 patients, $28 \mathrm{had}$ TNBC, and their age was $38-63$ years, with average age of $52.3 \pm 8.1$ years. And 32 patients had HER $2+B C$, and their age was 31-83 years, with average age of $44.6 \pm 7.6$ years. All patients had not received any treatment before surgery.

\section{Examination and diagnosis methods} ACUSON S2000 color Doppler ultrasound diagnostic instrument with $1415 \mathrm{BV}$ linear array probe (frequency 5-14 MHz) and corresponding ABVS system (Shanghai Siemens Medical Devices Co., Ltd., Shanghai, China) were used for the examination and diagnosis of breasts. First, the two-dimensional ultrasound was used to scan the fully exposed breasts. The virtual touch quantification (VTQ) mode of acoustic radiation force impulse (ARFI), a new generation of SWE, was selected. The shear wave velocity (SWV, $\mathrm{m} / \mathrm{s}$ ) at the edge of four quadrants of mass was obtained. The $S W V_{\text {max }}, S W V_{\text {min }}$, and $S W V_{\text {mean }}$ values were recorded. Then, the ABVS mode was selected to scan the breasts at anteroposterior, lateral (or medial), upper, and lower positions. The three-dimensional images of transverse, sagittal, and coronal planes were obtained by workstation $^{10}$. All imaging data were diagnosed by two doctors with 10 years of experience in breast ultrasound diagnosis using double-blind method. The coronary imaging, microcalcification, shape, edge, and other features of mass were analyzed.

\section{Statistical analysis}

SPSS version 19.0 statistical software (SPSS Inc., Chicago, IL, USA) was used for statistical analysis. The enumeration data were expressed as number and rate, and the comparison between groups was performed using $\chi^{2}$ test. The statistically significant parameters were analyzed by multivariate logistic regression. The measurement data are expressed as mean \pm standard deviation, and the comparison between groups was performed using t-test. Using immunohistochemistry result as gold standard, the diagnostic efficiencies of ABVS and ABVS+VTQ were analyzed using receiver operating characteristic curve, and the area under curve (AUC) among three methods was compared. A $p<0.05$ was considered statistically significant.

\section{RESULTS}

\section{Automated breast volume scanning imaging characteristics of triple-negative breast cancer and human epidermal growth factor receptor 2-positive breast cancer}

The single factor analysis of ABVS characteristics of patients in TNBC and HER $2+B C$ groups showed that there was no significant difference in coronal imaging or internal echo between TNBC and HER $2+B C$ groups ( $>0.05)$. The microcalcification, posterior echo, internal echo, shape, and edge had significant difference between TNBC and HER $2+B C$ groups $(p<0.05)$ (Table 1). Multivariate logistic regression analysis showed that the regular shape was the independent factor for TNBC $(\mathrm{p}=0.04$, odds ratio $[\mathrm{OR}]=4.479)$ and the microcalcification in mass for HER $2+B C(p=0.01, O R=2.997)$. The ABVS images of TNBC and HER2+BC are shown in Figure 1.

Shear wave elastography imaging characteristics of triple-negative breast cancer and human epidermal growth factor receptor 2-positive breast cancer

The VTQ of ARFI showed that the $S W V_{\text {max }}, S W V_{\text {min }}$, and $S W V_{\text {mean }}$ in TNBC group were 5.2 $\pm 0.5,3.7 \pm 0.7$, and $4.4 \pm 0.4$ $\mathrm{m} / \mathrm{s}$, respectively. The $S W V_{\text {max }}, S W V_{\text {min }}$, and $S W V_{\text {mean }}$ in HER2+BC group were $6.4 \pm 0.8,4.9 \pm 1.0$, and $5.7 \pm 0.8 \mathrm{~m} / \mathrm{s}$, respectively. Each index in TNBC group was significantly lower than that in HER2+BC group $\left(\mathrm{SWV}_{\max }: \mathrm{t}=6.032, \mathrm{p}<0.001\right.$; $\left.S W_{\text {min }}: t=4.950, p<0.001 ; W W V_{\text {mean }}: t=6.991, p<0.001\right)$.

\section{Diagnostic efficiency of automated breast} volume scanning and automated breast volume scanning+virtual touch quantification for triple-negative breast cancer

In diagnosing TNBC, the AUC of ABVS and ABVS+VTQ was $0.717,0.632$, and 0.768 , respectively. The AUC of ABVS+VTQ was larger than that of ABVS. The sensitivity, specificity, and accuracy 
Table 1. Single factor analysis of automated breast volume scanning characteristics of triple-negative breast cancer and human epidermal growth factor receptor 2-positive breast cancer.

\begin{tabular}{|c|c|c|c|c|}
\hline Characteristics & TNBC group & HER2+BC group & $\chi^{2}$ & $p$ \\
\hline N & 28 & 32 & & \\
\hline \multicolumn{5}{|l|}{ Coronal imaging, n (\%) } \\
\hline Convergence sign & $0(0.00)$ & $3(9.37)$ & \multirow{3}{*}{4.117} & \multirow{3}{*}{0.128} \\
\hline Hyperechoic halo & $2(7.14)$ & $5(15.63)$ & & \\
\hline Unchanged & $26(92.86)$ & $24(75.00)$ & & \\
\hline \multicolumn{5}{|l|}{ Microcalcification, n (\%) } \\
\hline Yes & $4(14.29)$ & $24(75.00)$ & \multirow{2}{*}{22.117} & \multirow{2}{*}{0.000} \\
\hline No & $24(85.71)$ & $8(25.00)$ & & \\
\hline \multicolumn{5}{|l|}{ Posterior echo, n (\%) } \\
\hline Enhance & $12(42.86)$ & $6(18.75)$ & \multirow{3}{*}{8.295} & \multirow{3}{*}{0.016} \\
\hline Sound and shadow & $1(3.57)$ & $9(28.13)$ & & \\
\hline No change & $15(53.57)$ & $17(53.12)$ & & \\
\hline \multicolumn{5}{|l|}{ Internal echo, n (\%) } \\
\hline Hypoechoic & $23(82.14)$ & $20(62.50)$ & \multirow{3}{*}{2.860} & \multirow{3}{*}{0.239} \\
\hline Isoechoic & $1(3.57)$ & $2(6.25)$ & & \\
\hline Inhomogeneous echo & $4(14.29)$ & $10(31.25)$ & & \\
\hline \multicolumn{5}{|l|}{ Shape, n (\%) } \\
\hline Regular (round/oval) & $18(64.29)$ & $5(15.63)$ & \multirow{2}{*}{14.959} & \multirow{2}{*}{0.000} \\
\hline Irregular & $10(35.71)$ & $27(84.37)$ & & \\
\hline \multicolumn{5}{|l|}{ Edge, n (\%) } \\
\hline Smoothing & $20(71.43)$ & $7(18.75)$ & \multirow{3}{*}{17.177} & \multirow{3}{*}{0.000} \\
\hline Microlobulation & $7(25.00)$ & $13(25.00)$ & & \\
\hline Angulation/burr & $1(3.57)$ & $12(56.25)$ & & \\
\hline
\end{tabular}

TNBC: triple-negative breast cancer; HER2+BC: human epidermal growth factor receptor 2-positive breast cancer.

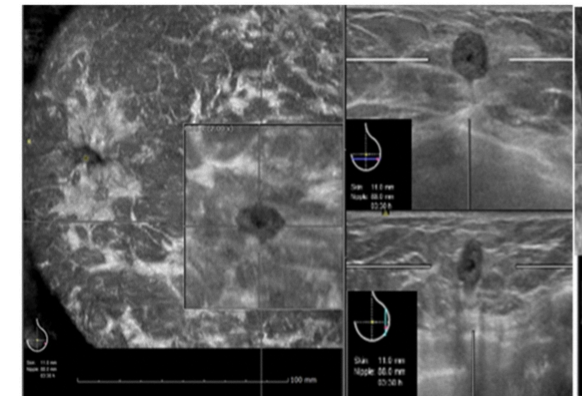

A

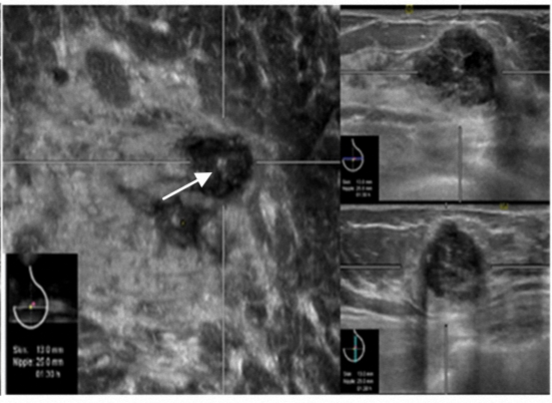

B

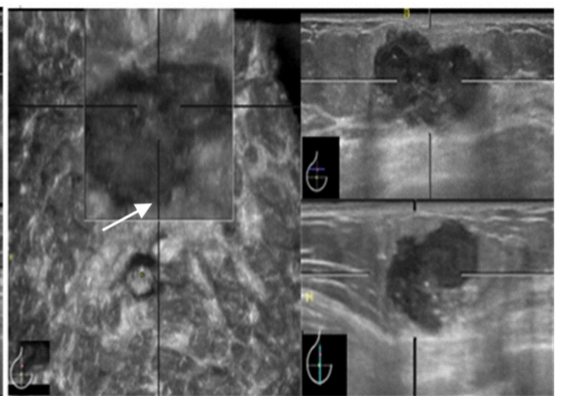

$\mathrm{C}$

Figure 1. (A) automated breast volume scanning image of triple-negative breast cancer. The shape of mass was regular, and there was no microcalcification. (B) automated breast volume scanning image of human epidermal growth factor receptor 2-positive breast cancer. There was obvious microcalcification in mass on the coronal plane (arrow). (C) automated breast volume scanning image of human epidermal growth factor receptor 2-positive breast cancer. There was obvious angulation on the edge of coronal plane and sagittal plane (arrow). 
of ABVS were 71.4, 71.9, and 70.5\%, respectively. The sensitivity, specificity and accuracy of ABVS+VTQ were 78.6, 75.4, and 75.0\%, respectively. Therefore, the sensitivity, specificity, and accuracy of $\mathrm{ABVS}+\mathrm{VTQ}$ were higher than those in ABVS alone (Figure 2).

\section{DISCUSSION}

Due to the standardization of scanning procedure, ABVS has better repeatability, higher consistency, and more comprehensive information than conventional two-dimensional ultrasound. It can display the differential resolutions of image and tissue anatomical characteristics and spatial relationship, which is of great significance in the localization and diagnosis of breast cancer ${ }^{11}$. The sagittal view of ABVS has advantage in observing the angulation in edge, the coronal view has advantage in observing the burr in edge, and the cross-sectional view has the highest resolution ${ }^{12}$. In the absence of mass background, ABVS has a higher detection rate of microcalcification than conventional ultrasound, which plays an important complementary role in the detection of microcalcification ${ }^{13}$.

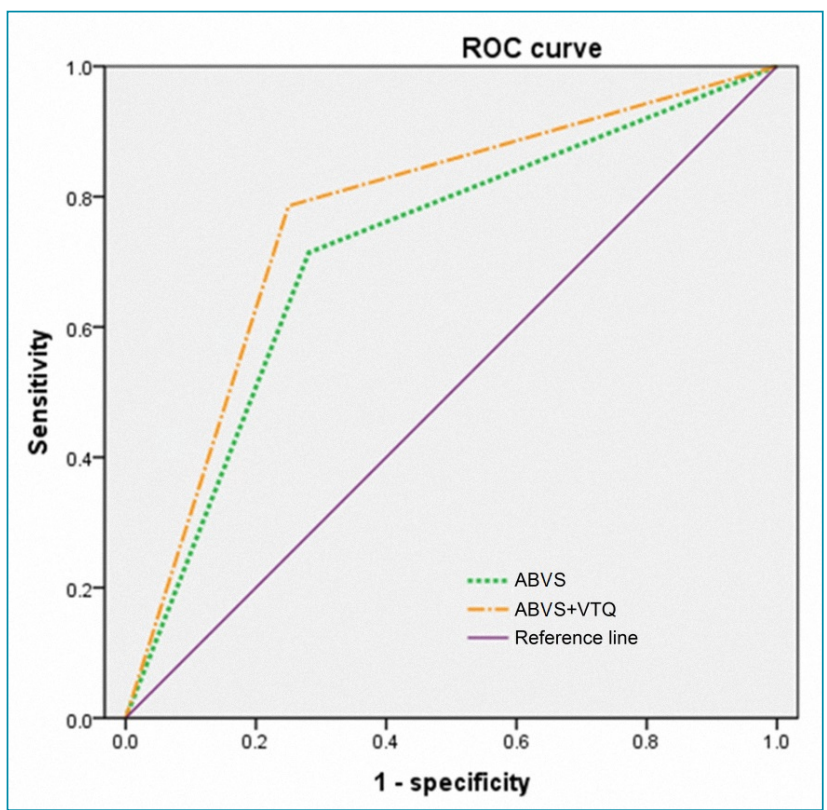

Figure 2. Receiver operating characteristic curves of automated breast volume scanning and automated breast volume scanning+virtual touch quantification in diagnosing triple-negative breast cancer.
Some scholars ${ }^{14}$ have studied the ABVS-related manifestations and proposed that the marginal burr sign of breast cancer is positively correlated with the positive expression of estrogen receptor and progesterone receptor and that the microcalcification in mass is positively correlated with HER2-positive expression. Both TNBC and HER2+BC have negative estrogen receptor and progesterone receptor expressions, but HER2+BC has positive HER2 expression. Results of this study showed that in TNBC and HER2+BC, the convergence sign of ABVS was rare and hyperechoic halo was not obvious. However, the microcalcification in mass was the independent factor for HER2+BC. This can be used for differentiation of HER2+BC from TNBC.

Results of our study indicate that SWE could provide the additional information for the quantitative diagnosis of TNBC and HER2+BC. In our study, the SWV max,$S W V_{\text {min }}$, and $S W V_{\text {mean }}$ values in TNBC group were significantly lower than those in HER2+BC group. ABVS+VTQ can show the relatively low stiffness of TNBC, so the sensitivity, specificity, and accuracy of ABVS+VTQ were higher than those in ABVS alone.

\section{CONCLUSIONS}

ABVS combined with SWE has certain advantages in differentiating TNBC from HER2+BC, which is helpful for the treatment planning and prognosis judgment. The limitation of this study is that, due to the relatively small sample size, the clinical tumor node metastasis stage of selected patients is not considered. Next, the sample size should be further increased, and the in-depth study should be conducted to put forward the advantages of the combined differential diagnosis method.

\section{ACKNOWLEDGMENTS}

This work was supported by the Major Scientific and Technological Project in Xiaoshan District (2017218).

\section{AUTHORS" CONTRIBUTIONS}

WC: Supervision, Validation, Writing - review \& editing. RR: Conceptualization, Funding acquisition, Investigation. FW: Data curation, Formal analysis, Software. ML: Methodology, Project administration, Resources, Writing - original draft.

\section{REFERENCES}

1. Gradishar WJ, Anderson BO, Balassanian R, Blair SL, Burstein $\mathrm{HJ}$, Cyr A, et al. NCCN guidelines insights: breast cancer, version 1.2017. J Natl Compr Canc Netw. 2017;15(4):433-51. https://doi.org/10.6004/jnccn.2017.0044
2. Zhang $M$, Zhang $X$, Zhao S, Wang $Y$, Di W, Zhao G, et al. Prognostic value of survivin and EGFR protein expression in triple-negative breast cancer (TNBC) patients. Target Oncol. 2014;9(4):349-57. https://doi.org/10.1007/s11523-013-0300-y 
3. Bonzanini M, Morelli L, Bonandini EM, Leonardi E, Pertile R, Palma PD. Cytologic features of triple-negative breast carcinoma. Cancer Cytopathol. 2012;120(6):401-9. https:// doi.org/10.1002/cncy.21207

4. Abouharb S, Ensor J, Loghin ME, Katz R, Moulder SL, Esteva FJ, et al. Leptomeningeal disease and breast cancer: the importance of tumor subtype. Breast Cancer Res Treat. 2014;146(3):47786. https://doi.org/10.1007/s10549-014-3054-z

5. Aksoy A, Odabas H, Kaya S, Bozkurt O, Degirmenci M, Topcu TO, et al. Hormone receptor status and survival of medullary breast cancer patients. A Turkish cohort. Saudi Med J. 2017;38(2):15662. https://doi.org/10.15537/smj.2017.2.18055

6. Van Zelst JCM, Platel B, Karssemeijer N, Mann RM. Multiplanar reconstructions of 3D automated breast ultrasound improve lesion differentiation by radiologists. Acad Radiol. 2015;22(12):1489-96. https://doi.org/10.1016/j. acra.2015.08.006

7. Mussetto I, Gristina L, Schiaffino S, Tosto S, Raviola E, Calabrese M. Breast ultrasound: automated or hand-held? Exploring patients' experience and preference. Eur Radiol Exp. 2020;4(1):12. https://doi.org/10.1186/s41747-019-0136-z

8. Taljanovic MS, Gimber LH, Becker GW, Latt LD, Klauser AS, Melville DM, et al. Shear-wave elastography: basic physics and musculoskeletal Applications. Radiographics. 2017;37(3):85570. https://doi.org/10.1148/rg.2017160116
9. Tamura M, Ohta H, Nisa K, Osuga T, Sasaki N, Morishita K, et al. Evaluation of liver and spleen stiffness of healthy dogs by use of two-dimensional shear wave elastography. Am J Vet Res. 2019;80(4):378-84. https://doi.org/10.2460/ajvr.80.4.378

10. Choi EJ, Choi H, Park EH, Song JS, Youk JH. Evaluation of an automated breast volume scanner according to the fifth edition of BI-RADS for breast ultrasound compared with hand-held ultrasound. Eur J Radiol. 2018;99:138-45. https:// doi.org/10.1016/j.ejrad.2018.01.002

11. Wenkel E, Heckmann M, Heinrich M, Schwab SA, Uder M, Schulz-Wendtland R, et al. Automated breast ultrasound: lesion detection and BI-RADS classification--a pilot study. Rofo. 2008;180(9):804-8. https://doi.org/10.1055/s-2008-1027563

12. Kaplan SS. Automated whole breast ultrasound. Radiol Clin North Am. 2014;52(3):539-46. https://doi.org/10.1016/j. rcl.2014.01.002

13. Chang RF, Huang SF, Wang LP, Chen DR, Moon WK. Microcalcification detection in 3-d breast ultrasound. Conf Proc IEEE Eng Med Biol Soc. 2005;2005:6297-300. https:// doi.org/10.1109/IEMBS.2005.1615937

14. Giuliano V, Giuliano C. Improved breast cancer detection in asymptomatic women using 3D-automated breast ultrasound in mammographically dense breasts. Clin Imaging. 2013;37(3):480-6. https://doi.org/10.1016/j. clinimag.2012.09.018 